\title{
Identification of Transfer Functions by a Method Based on Feedback System Theory
}

\author{
Kuniharu Kishida and Satoshi Masuda \\ Department of Information Science, Faculty of Engineering \\ Gifu University \\ Yanagido, Gifu 501-1193, JAPAN \\ kishida@cc.gifu-u.ac.jp
}

\begin{abstract}
From the viewpoint of stochastic inverse problem, identification of transfer functions in feedback systems has been examined by the previous papers[1][2], and applied to EEG analysis[3]. Some transfer functions between measurement regions are identified by our method of inverse problem in a stochastic feedback system. However, there are still open problems of our method for stochastic inverse problem: One problem is that there occurs a chaos-like behavior in matrix Riccati equation and it affects a property of identified innovation model. The other is a filtering effect on transfer functions for pre-processing of high pass filter. These problems are discussed from the viewpoint of statistical inverse problem.
\end{abstract}

\section{Introduction}

This is the forth paper in a series of modeling of stochastic feedback systems, in which we hope to develop an identification method of transfer functions in complex systems from the viewpoint of inverse problems. When we apply our method to the EEG analysis, some transfer functions between measurement regions have been identified as mentioned in [3]. However, there is an open problem of our method. A chaos-like behavior has happened to occur in Riccati equation of our formalism and then affect a property of identified innovation model. It is important to examine an origin of the behavior. From the viewpoint of statistical inverse problems it will be discussed that such a phenomenon occurs under some situations [4] due to a joint effect between statistical errors and nonlinearity of Eq. (3). High pass filter (HPF) effects on identification of transfer functions will be also examined, since HPFs are often used to remove artifacts of eye movements in EEG time series data.

\section{Identification method based on feedback system theory}

If a stochastic feedback system between measurement variables can be found in a Gaussian stationary process, we can identify a feedback model with transfer functions $F_{i j}\left(z^{-1}\right)$ between measurement variables $y_{i}(n)$ :

$$
\begin{aligned}
& y_{1}(n)=F_{12}\left(z^{-1}\right) y_{2}(n)+F_{1}\left(z^{-1}\right) \gamma_{1}(n) \\
& y_{2}(n)=F_{21}\left(z^{-1}\right) y_{1}(n)+F_{2}\left(z^{-1}\right) \gamma_{2}(n)
\end{aligned}
$$

where $z^{-1}$ is the backward time shift operator: $z^{-1} y_{i}(n)=y_{i}(n-1)$. Four transfer functions of the feedback model are given as

$$
\begin{aligned}
F_{i j}\left(z^{-1}\right) & :=C_{i j}\left(I-A_{i j} z^{-1}\right)^{-1} B_{i j} z^{-1} \\
F_{i}\left(z^{-1}\right) & :=C_{i}\left(I-A_{i} z^{-1}\right)^{-1} B_{i} .
\end{aligned}
$$

The feedback model describes one of subsystems included in a total system as in [3]. Some of transfer functions between measurement variables of subsystem can be identified in our method of inverse problem, and an innovation model has a minimum phase property, which is equivalent to the original stochastic feedback system in terms of correlation functions. To obtain a numerical innovation model of Eq. (1) as mentioned in the book [5], procedures of our formalism for a stationary Gaussian time series data are summarized as five steps:

Step 1: Calculate correlation function matrices $R_{y y}(k)$ from $N$ time series data, $(y(n), n=1,2, \ldots, N)$ : $R_{y y}(k)=\frac{1}{N-k} \sum_{j=1}^{N-k}\left(y(j+k) y(j)^{T}\right)$, where the mean is assumed to be zero: $E\{y(n)\}=\frac{1}{N} \sum_{j=1}^{N} y(j)=0$.

Step 2: Define a Hankel matrix $H_{y y}(k)$, and its associate matrices, $H_{A}(k), H_{B}(k)$ and $H_{C}(k)$ as

$$
\begin{gathered}
H_{y y}(k):=\left(\begin{array}{cccc}
R_{y y}(0) & R_{y y}(1) & \ldots & R_{y y}(k) \\
R_{y y}(1) & R_{y y}(2) & \ldots & R_{y y}(k+1) \\
\vdots & \vdots & & \vdots \\
R_{y y}(k) & R_{y y}(k+1) & \ldots & R_{y y}(2 k)
\end{array}\right) \\
H_{A}(k):=\left(\begin{array}{cccc}
R_{y y}(1) & R_{y y}(2) & \ldots & R_{y y}(k+1) \\
R_{y y}(2) & R_{y y}(3) & \ldots & R_{y y}(k+2) \\
\vdots & \vdots & & \vdots \\
R_{y y}(k+1) & R_{y y}(k+2) & \ldots & R_{y y}(2 k+1)
\end{array}\right) \\
H_{B}(k):=\left(\begin{array}{c}
R_{y y}(0) \\
R_{y y}(1) \\
\vdots \\
R_{y y}(k)
\end{array}\right)
\end{gathered}
$$


$H_{C}(k):=\left(\begin{array}{llll}R_{y y}(0) & R_{y y}(1) & \ldots & R_{y y}(k)\end{array}\right)$.

Step 3: Calculate three matrices $A, B_{q}$ and $C$ from the associated matrices, $H_{A}(k), H_{B}(k)$, and $H_{C}(k)$ by using the singular value decomposition of the Hankel matrix $H_{y y}(k)$. That is, we have

$$
\begin{gathered}
A=\Sigma_{k}^{-1 / 2} U_{k}^{T} H_{A}(k) V_{k} \Sigma_{k}^{-1 / 2} \\
B_{q}=\Sigma_{k}^{-1 / 2} U_{k}^{T} H_{B}(k) \\
C=H_{C}(k) V_{k} \Sigma_{k}^{-1 / 2},
\end{gathered}
$$

where $H_{y y}(k)=U_{k} \Sigma_{k} V_{k}^{T}, \Sigma_{k}$ is a diagonal matrix of singular values and $U_{k}$ and $V_{k}$ are orthogonal matrices.

Step 4: A stable solution of Riccati equation with $A, B_{q}$ and $C$ can be calculated by iteration procedure,

$$
E(n+1)=A E(n) A^{T}+A L(n)\left(B_{q}-E(n) C^{T}\right)^{T} A^{T} .
$$

where $L(n):=\left(B_{q}-E(n) C^{T}\right)\left(R_{y y}(0)-C E(n) C^{T}\right)^{\dagger}$. A matrix $B$ is defined from a stable solution $E(\infty)$ of Eq. (3): $B=\left(B_{q}-E(\infty) C^{T}\right)\left(R_{y y}(0)-C E(\infty) C^{T}\right)^{\dagger}$. After we have obtained the stable solution, we can go to the next step 5 of our formalism.

Step 5: We have a numerical innovation model,

$$
\begin{aligned}
x(n \mid n) & =A x(n-1 \mid n-1)+B \gamma(n) \\
y(n) & =C x(n \mid n) .
\end{aligned}
$$

Coefficient matrices $A, B$ and $C$ of the numerical innovation model should have minimum phase properties of pole stability and zero invertibility

$$
\operatorname{eig}|A|<1 \quad \text { and } \operatorname{eig}|A(I-B C)|<1 .
$$

and the consistence of the innovation model

$$
C B=I \rightarrow \operatorname{det}(C B)=1 .
$$

These conditions are good criteria for identification of numerical innovation model.

For a numerical innovation model, Eq. (4), we have

$$
\hat{G}\left(z^{-1}\right)=C\left(I-A z^{-1}\right)^{-1} B,
$$

where $\hat{G}\left(z^{-1}\right) \Rightarrow G^{\gamma}\left(z^{-1}\right)$ as the number of time series data becomes large. Here, a closed loop transfer function matrix of a theoretical innovation model can be determined as

$$
G^{\gamma}\left(z^{-1}\right):=H\left(I-\Phi z^{-1}\right)^{-1} K,
$$

where matrices $H, \Phi$ and $K$ are coefficient ones of the theoretical innovation model equivalent to the stochastic system as in [5]. Open loop transfer functions can be obtained by using a transformation of feedback structure from closed loop transfer functions as in [5]. There are sufficient conditions to identify open loop transfer functions $F_{i j}\left(z^{-1}\right)$ : In the case of square problem, Kishida has showed that open loop transfer functions are identified under two sufficient conditions of minimum phase and independence of random forces, which are similar to results mentioned by Goodwin and Payne [6]. In the case of overdeterminant problem, Kishida has showed in [1] that $F_{i j}\left(z^{-1}\right)$ can be identified under the generalized minimum phase condition. In the case of underdetermined problem Kishida and Sato have discussed that $F_{i j}\left(z^{-1}\right)$ are not identified exactly but almost correctly [2]. Under such a suitable condition in which the feedback structure is conserved, open loop transfer functions are determined by a transformation from closed loop transfer functions of Eq. (4):

$$
\begin{aligned}
& \hat{F}_{12}\left(z^{-1}\right)=G_{12}\left(z^{-1}\right) G_{22}\left(z^{-1}\right)^{-1} \\
& \hat{F}_{21}\left(z^{-1}\right)=G_{21}\left(z^{-1}\right) G_{11}\left(z^{-1}\right)^{-1} .
\end{aligned}
$$

Finally a model reduction algorithm of open loop transfer functions is needed to evaluate the original open loop transfer functions.

When our formalism was applied to the EEG analysis [3], the convergence of $E(n)$ of Eq.(3) was almost monotonic, and was examined by a condition $\left|\left(E_{i, j}(n+1)-E_{i, j}(n)\right) / E_{i, j}(n)\right|<0.00001$. We marked such a case by the symbol "o" in Figure 1, if the innovation model was of minimum phase. Figure 1 shows whether innovation models between EEG channels are minimum phase or not. The symbol "*" means that

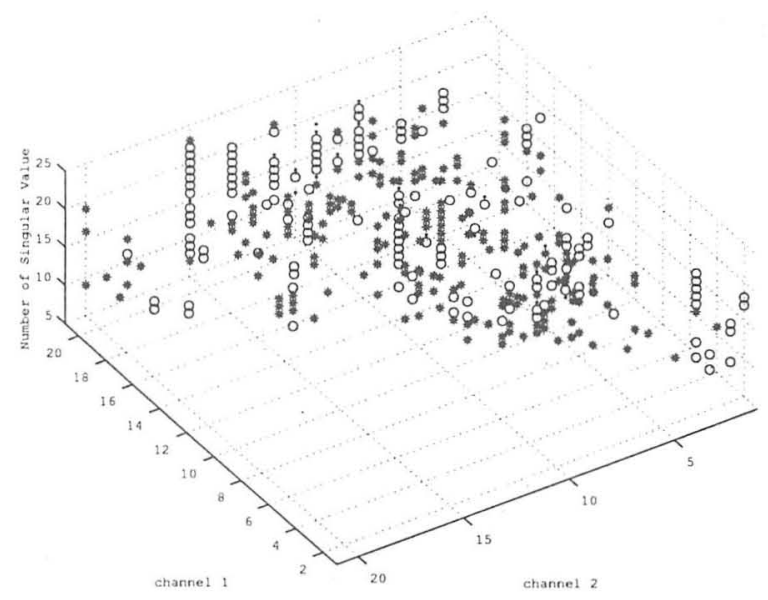

Figure 1: minimum phase innovation model of "imam" between two selected channels among 21 channels

iteration of Riccati equation, Eq. (3), was terminated forcedly at a maximum value and that the innovation model was minimum phase as will be shown in the cases of Figures 3 and 5. Such a solution may be considered to be convergent, if the point of the maximum value is far from thorns. There is a possibility that we can have almost correct transfer functions even in such a chaotic case, where the above mentioned "chaotic" behavior is seen in Eq.(3). Therefore, there remains an open problem of thorns in $E(n)$ in our identification method. To know the origin of "thorns" in Riccati equation we will 
examine a simulation study similar to EEGs in the following sections.

\section{An simple example and chaotic behavior}

Let us consider a linear Gaussian part given by a 2input and 2-output feedback system:

$$
\begin{aligned}
y_{1}(n) & =\frac{-0.36 z^{-1}}{1-0.89 z^{-1}} y_{2}(n)+\frac{0.21}{1-0.75 z-1} f_{1}(n) \\
y_{2}(n) & =\frac{0.15 z^{-1}}{1-0.98 z^{-1}} y_{1}(n)+\frac{0.34}{1-0.8 z^{-1}} f_{2}(n)
\end{aligned}
$$

It is assumed that $f_{1}(n)$ and $f_{2}(n)$ are independent, white and Gaussian, and that their covariance is unit matrix. The feedback system has four poles, $(0.935 \pm$ $0.22798 i)^{-1}, 0.75^{-1}$, and $0.8^{-1}$ in the $z^{-1}$ plane. The feedback system is stable, since all poles are outside the unit circle in the $z^{-1}$ plane or inside the unit circle in the $\mathrm{z}$ plane. Two original zeros of the feedback system are invertible $0.89^{-1}$ and $0.98^{-1}$. Since all poles and zeros are stable and invertible, the feedback system is in a minimum phase. From Eq. (9) we can estimate theoretically open loop transfer functions as

$$
\begin{aligned}
\hat{F}_{12}\left(z^{-1}\right) & =G_{12}^{\gamma}\left(z^{-1}\right) G_{22}^{\gamma}\left(z^{-1}\right)^{-1} \\
& =\frac{-0.36 z^{-1}}{1-0.89 z^{-1}}=F_{12}\left(z^{-1}\right) \\
\hat{F}_{21}\left(z^{-1}\right) & =G_{21}^{\gamma}\left(z^{-1}\right) G_{11}^{\gamma}\left(z^{-1}\right)^{-1} \\
& =\frac{0.15 z^{-1}}{1-0.98 z^{-1}}=F_{21}\left(z^{-1}\right) .
\end{aligned}
$$

\subsection{Example of infinite time series data}

From coefficient matrices $\Phi, G, H$ and $V$ corresponding to Eq. (10), correlation functions of infinite number of time series data can be evaluated theoretically [5] as

$$
C_{y y}(n)=H \Phi^{n} C_{x x}(0) H^{T},
$$

where $C_{x x}(0)$ is calculated from the Lyapunov equation:

$$
C_{x x}(0)=\Phi C_{x x}(0) \Phi^{T}+G V G^{T} .
$$

Therefore, the closed loop transfer function matrix via coefficient matrices $A, B$ and $C$ of Eq. (4) is given from $C_{y y}(n)$ as

$$
\hat{G}\left(z^{-1}\right)=C\left(I-A z^{-1}\right)^{-1} B=G^{\gamma}\left(z^{-1}\right) .
$$

We have estimated open loop transfer functions,

$$
\begin{aligned}
\hat{F}_{12}\left(z^{-1}\right) & =\hat{G}_{12}\left(z^{-1}\right) \hat{G}_{22}\left(z^{-1}\right)^{-1} \\
& =\frac{-2.343 \cdot 10^{-14}-0.36 z^{-1}}{1-0.89 z^{-1}} \\
\hat{F}_{21}\left(z^{-1}\right) & =\hat{G}_{21}\left(z^{-1}\right) \hat{G}_{11}\left(z^{-1}\right)^{-1} \\
& =\frac{-1.027 \cdot 10^{-15}+0.15 z^{-1}}{1-0.98 z^{-1}} .
\end{aligned}
$$

If round errors are ignored in Eq. (13), we can identify the original open loop transfer functions $F_{12}\left(z^{-1}\right)$ and $F_{21}\left(z^{-1}\right)$. We also have $C B=I$. Let us examine hereafter effects on identification of open loop transfer functions owing to statistical errors.

\subsection{Satisfactory example with 15000 time series data}

Statistical quantities of $y_{1}$ and $y_{2}$ depend on the total number of time series data. Estimated correlation functions $R_{y y}(n)$ for $\mathrm{N}=15000$ are expected to be convergent to theoretical ones $C_{y y}(n)$. When Eq. (3) is repeated 1000 times, the $(2,3)$ th element of $E(n)$, $E_{2,3}(n)$, approaches monotonously to a constant solution as shown in Figure 2. Then, we understand that

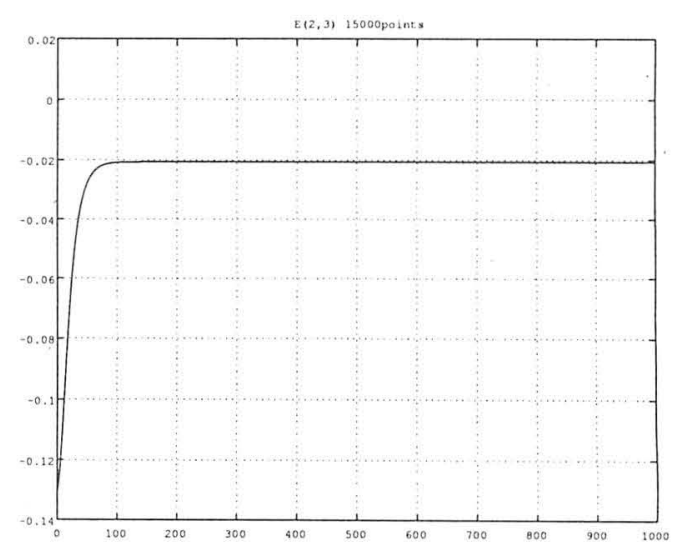

Figure 2: usual convergence of Riccati equation

a matrix solution of Eq. (3) approaches to a fixed point of $E(\infty)$, which corresponds to a stable solution. Coefficient matrices of numerical innovation model for $\mathrm{N}=15000$ give a closed loop transfer function matrix,

$$
\hat{G}\left(z^{-1}\right)=C\left(I-A z^{-1}\right)^{-1} B,
$$

and a matrix product $C B$ becomes almost an unit matrix:

$$
C B=\left(\begin{array}{ll}
1.01648 & -0.00255 \\
0.02023 & 0.983396
\end{array}\right) .
$$

Estimated open loop transfer functions are evaluated as

$$
\begin{aligned}
\hat{F}_{12}\left(z^{-1}\right) & =\hat{G}_{12}\left(z^{-1}\right) \hat{G}_{22}\left(z^{-1}\right)^{-1} \\
& =\frac{-0.00259-0.3627 z^{-1}}{1-0.8889 z^{-1}} \simeq F_{12}\left(z^{-1}\right) \\
\hat{F}_{21}\left(z^{-1}\right) & =\hat{G}_{21}\left(z^{-1}\right) \hat{G}_{11}\left(z^{-1}\right)^{-1} \\
& =\frac{0.0199+0.124 z^{-1}}{1-0.9797 z^{-1}} \simeq F_{21}\left(z^{-1}\right) .
\end{aligned}
$$

From this result it is concluded that $N=15000$ is large enough to identify open loop transfer functions. If the number of time series data is not enough, we will have a chaos-like behavior of $E(n)$ in Eq. (3) in the following subsection. 


\subsection{Unsatisfactory example with 1500 time series data}

When the total number of time series data is not large enough, we can show an unsatisfactory example with $\mathrm{N}=1500$. That is, there occurs a trouble due to statistical errors in our formalism in determination of an innovation model from correlation functions: Numerical evaluations by simulation were not satisfactory, since non-diagonal elements of $C B$ are not small:

$$
C B=\left(\begin{array}{cc}
0.8910696 & -0.0072774 \\
0.17346962 & 0.9375564
\end{array}\right)
$$

We have checked iteration behavior of $E_{2,3}(n)$, and found that $E(n)$ does not converge into a fixed point as in Figure 3. We have thorns in $E(n)$ of Figure 3. Therefore, we could not have a stable solution of Riccati equation. Since iteration evolution of the solution of Riccati

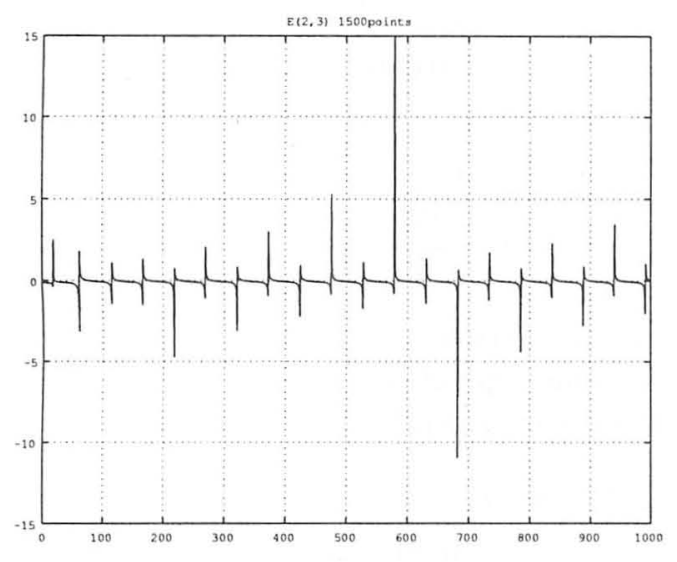

Figure 3: chaos-like behavior in Riccati equation

equation seems to be "chaotic", we have examined a return map of $E_{2,3}(n)$ and found that the return map is a nonlinear function as in Figure 4. Here the symbol of

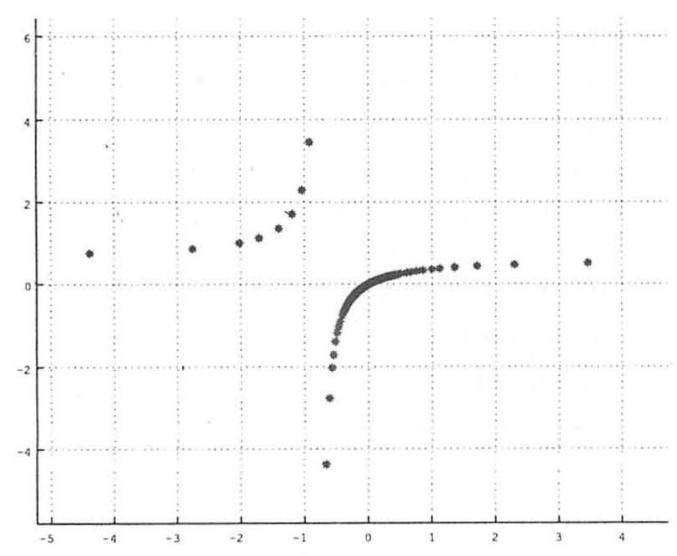

Figure 4: return map of $E_{2,3}(n)$

* means a position of $\left(E_{2,3}(n), E_{2,3}(n+1)\right)$. Therefore, the solution of Riccati equation is chaos-like.

To know the origin of chaos-like phenomenon, we have examined a matrix of $Q(n):=\left(R_{y y}(0)-C E(n) C^{T}\right)$. $Q(n)$ must be positive definite from its definition as in [7]. However, in the simulation there occurs a case where $Q(n)$ at $n=218$ becomes negative definite as in the followings

$$
\begin{array}{r}
Q(216)=\left(\begin{array}{cc}
0.05464413034475 & -0.00099553205355 \\
-0.00099553205355 & 0.11657354703003
\end{array}\right) \\
\operatorname{det}(Q(216))=0.00636906901459 \\
Q(217)=\left(\begin{array}{cc}
0.02573997316787 & -0.03389814667175 \\
-0.03389814667175 & 0.06949914990794
\end{array}\right) \\
\operatorname{det}(Q(217))=0.00063982190604 \\
Q(218)=\left(\begin{array}{ll}
-0.11235827214040 & -0.22364005658663 \\
-0.22364005658663 & -0.19119925500206
\end{array}\right) \\
\operatorname{det}(Q(218))=-0.02853205698351 \\
Q(219)=\left(\begin{array}{ll}
0.07365282465281 & 0.03193196086317 \\
0.03193196086317 & 0.15994676457943
\end{array}\right) \\
\operatorname{det}(Q(219))=0.01076088088079
\end{array}
$$

This negative definite occurs owing to statistical errors of correlation functions in $Q(n)$ and gives thorns in iteration evolution of $E(n)$. By iteration procedure of Riccati equation, we have not a stable constant matrix but a chaos-like solution as similar to Figure 3. However, it should not be said that the thorn phenomenon is chaotic before examination of its Lyapunov exponent.

Coefficient matrices of Eq. (4) for $\mathrm{N}=1500$ give a closed loop transfer function matrix, and estimated open loop transfer functions are determined from our formalism as

$$
\begin{aligned}
\hat{F}_{12}\left(z^{-1}\right) & =\hat{G}_{12}\left(z^{-1}\right) \hat{G}_{22}\left(z^{-1}\right)^{-1} \\
& =\frac{-0.007762-0.3509 z^{-1}}{1-0.8886 z^{-1}} \sim F_{12}\left(z^{-1}\right) \\
\hat{F}_{21}\left(z^{-1}\right) & =\hat{G}_{21}\left(z^{-1}\right) \hat{G}_{11}\left(z^{-1}\right)^{-1} \\
& =\frac{0.1947+0.009087 z^{-1}}{1-1.084 z^{-1}} \neq F_{21}\left(z^{-1}\right) .
\end{aligned}
$$

Considerable differences are found in poles and zeros between the numerical innovation model for $N=1500$ and the theoretical innovation model. This unsatisfactory example is due to statistical errors in estimated correlations functions and parameters of Eq. (10). It is concluded that the chaos-like solution of Riccati equation gives unsatisfactory identification of open loop transfer functions in the above situation of our formalism.

\section{Some examples}

Let us examine other cases similar to the feedback model. We treat a pole of $F_{21}$ in Eq. (10) as an or- 
der parameter $\beta$ in the same feedback model as

$$
\begin{array}{r}
y_{1}(n)=\frac{-0.36 z^{-1}}{1-0.89 z^{-1}} y_{2}(n)+\frac{0.21}{1-0.75 z-1} f_{1}(n) \\
y_{2}(n)=\frac{0.15 z^{-1}}{1-\beta z^{-1}} y_{1}(n)+\frac{0.34}{1-0.8 z^{-1}} f_{2}(n) .
\end{array}
$$

The others are the same as Eq. (10).

\subsection{Linear stability of Eq. (17)}

From linear stability of $x^{2}-(0.89+\beta) x+0.89 \beta+0.36 *$ $0.15=0$, we have three conditions:

$$
\begin{gathered}
|0.89 \beta+0.054|<1 \Longleftrightarrow-1.184<\beta<1.0629 \\
1-\beta-0.89+0.89 \beta+0.054>0 \Longleftrightarrow \beta<1.4909 \\
1+\beta+0.89+0.89 \beta+0.054>0 \Longleftrightarrow \beta>-1.02857
\end{gathered}
$$

Then we have a stable region of the feedback model with order parameter $\beta$ : $-1.02857<\beta<1.0629$. And $-1<$ $\beta<1$ is invertible of zeros. Therefore, the feedback model with $1 \leq \beta<1.0629$ or $-1.02857<\beta \leq-1$ is in a nonminimum phase, and that with $-1<\bar{\beta}<1$ is in a minimum phase.

\subsection{Cases of $\beta=1$ and 1.02}

From sufficient condition of independence of random forces open loop transfer functions can be identified correctly in cases of $\beta=1$ and 1.02, though the feedback model with $\beta=1$ or 1.02 is not in a minimum phase. Let us consider a nonminimum phase case of $\beta=1.02$ and the total number of time series data be $N=20000$. From the independence of noise force we can identify open loop transfer functions [5] as

$$
\begin{aligned}
\hat{F}_{12}\left(z^{-1}\right) & =\hat{G}_{12}\left(z^{-1}\right) \hat{G}_{22}\left(z^{-1}\right)^{-1} \\
& =\frac{0.007545-0.373 z^{-1}}{1-0.8853 z^{-1}} \simeq F_{12}\left(z^{-1}\right) \\
\hat{F}_{21}\left(z^{-1}\right) & =\hat{G}_{21}\left(z^{-1}\right) \hat{G}_{11}\left(z^{-1}\right)^{-1} \\
& =\frac{-0.01145+0.1771 z^{-1}}{1-1.016 z^{-1}} \simeq F_{21}\left(z^{-1}\right)
\end{aligned}
$$

If the number of time series data becomes $N=1500$, we have a chaos-like solution of $E(n)$ as in Figure 5. And identified open loop transfer functions are not good:

$$
\begin{aligned}
\hat{F}_{12}\left(z^{-1}\right) & =\hat{G}_{12}\left(z^{-1}\right) \hat{G}_{22}\left(z^{-1}\right)^{-1} \\
& =\frac{-0.06199-0.2755 z^{-1}}{1-1.005 z^{-1}} \neq F_{12}\left(z^{-1}\right) \\
\hat{F}_{21}\left(z^{-1}\right) & =\hat{G}_{21}\left(z^{-1}\right) \hat{G}_{11}\left(z^{-1}\right)^{-1} \\
& =\frac{0.2048-0.1202 z^{-1}}{1-0.9863 z^{-1}} \neq F_{21}\left(z^{-1}\right) .
\end{aligned}
$$

For the case of $\beta=1$ and $N=2000$, we have the same

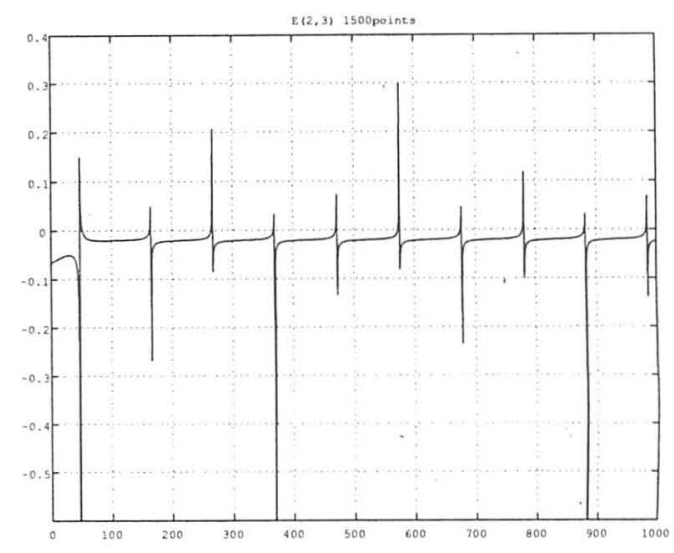

Figure 5: chaos-like behavior of $E_{2,3}(n)$ for $\beta=1.02$

chaos-like solution of $E(n)$ as in Figure 5, and have

$$
\begin{aligned}
\hat{F}_{12}\left(z^{-1}\right) & =\hat{G}_{12}\left(z^{-1}\right) \hat{G}_{22}\left(z^{-1}\right)^{-1} \\
& =\frac{-0.06046-0.3592 z^{-1}}{1-1.085 z^{-1}} \neq F_{12}\left(z^{-1}\right) \\
\hat{F}_{21}\left(z^{-1}\right) & =\hat{G}_{21}\left(z^{-1}\right) \hat{G}_{11}\left(z^{-1}\right)^{-1} \\
& =\frac{0.2519-0.2569 z^{-1}}{1-1.041 z^{-1}} \neq F_{21}\left(z^{-1}\right) .
\end{aligned}
$$

If the number of time series data becomes $N=20000$, we have the same type of convergence of $E(n)$ as Figure 2, and have correct open loop transfer functions

$$
\begin{aligned}
\hat{F}_{12}\left(z^{-1}\right) & =\hat{G}_{12}\left(z^{-1}\right) \hat{G}_{22}\left(z^{-1}\right)^{-1} \\
& =\frac{-0.004753-0.3568 z^{-1}}{1-0.896 z^{-1}} \simeq F_{12}\left(z^{-1}\right) \\
\hat{F}_{21}\left(z^{-1}\right) & =\hat{G}_{21}\left(z^{-1}\right) \hat{G}_{11}\left(z^{-1}\right)^{-1} \\
& =\frac{0.0113+0.1351 z^{-1}}{1-0.9762 z^{-1}} \sim F_{21}\left(z^{-1}\right) .
\end{aligned}
$$

\section{Effects of high pass filter}

Since a HPF is often used to remove artifacts of eye movements in the EEG/MEG analysis, it is important to examine HPF effects in identification of transfer functions. In the present section we examine it by a simulation study. To evaluate numerical HPF effects in the EEG analysis by our identification of transfer functions, we have used a feedback simulation model with Gaussian independent noise forces and four transfer functions in Eq. (1); one is an EEG type, $F_{12}\left(z^{-1}\right)=$ $\frac{0.1 z^{-1}-0.0866 z^{-2}+0.025849 z^{-3}-0.0024077 z^{-4}-0.0002479 z^{-5}}{1-3.61045 z^{-1}+5.44796 z^{-2}-4.19499 z^{-3}+1.5509 z^{-4}-0.17754 z^{-5}}$, and three simple ones $F_{21}\left(z^{-1}\right)=\frac{0.15 z^{-1}}{1-0.1 z^{-1}}, F_{1}\left(z^{-1}\right)=$ $\frac{1}{1-0.6 z^{-1}}$, and $F_{2}\left(z^{-1}\right)=\frac{1}{1-0.5 z^{-1}}$. The convergence condition of $E(n)$ in our formalism is the same as mentioned in section 2 .

We have used a butterworth type of zero-phase digital HPF of degree $7, H_{7}\left(z^{-1}\right)$, and obtained filtered time 
series data: $w_{i}(n)=H_{7}\left(z^{-1}\right) y_{i}(n)(\mathrm{i}=1,2)$. The number of time series data was 100000 to examine HPF effects exactly. Correlation matrices $R_{w w}(n)$ are input data for our identification formalism, and identified open loop transfer functions are shown in Figure 6. In
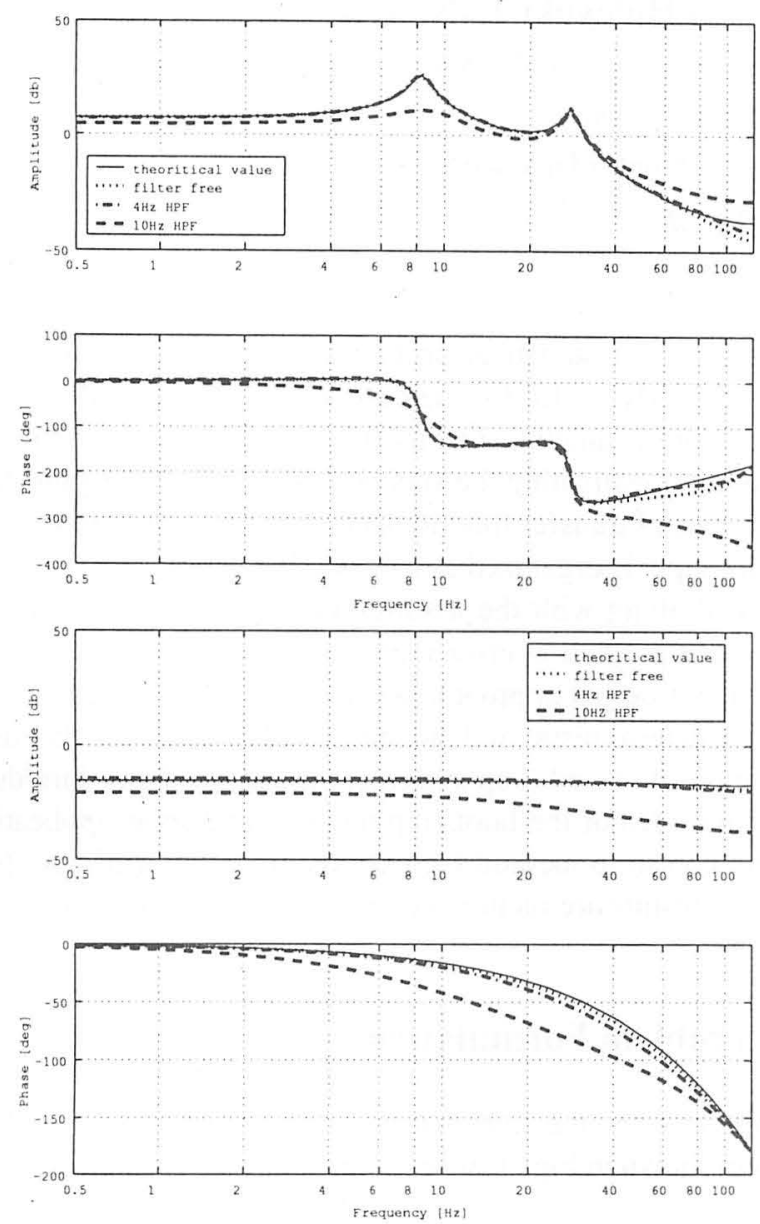

Figure 6: Transfer Functions $F_{12}$ (up) and $F_{21}$ (down)

Figure 6 , a solid line is the bode diagram of the original transfer functions, and dotted, dashdot and dashed lines mean the cases of filter free, HPF with the $4 \mathrm{~Hz}$ and $10 \mathrm{~Hz}$ corner frequency, respectively. It is concluded that in a high pass filter with lower than $5 \mathrm{~Hz}$ cutoff frequency open loop transfer functions are identified almost correctly in our formalism.

\section{Conclusion and remarks}

From the viewpoint of statistical inverse problems it is discussed by simulation study that in iteration procedure of Riccati equation of our identification method there are chaos-like solutions as similar to Figures 3 and 5. It is concluded that such a thorn-phenomenon occurs under some situations owing to a joint effect between statistical errors and nonlinearity of Riccati equation, Eq. (3). To identify transfer functions correctly it is necessary to avoid cases of the chaotic behavior in our identification method based on feedback system theory. Then, the chaos-like solution of Riccati equation gives unsatisfactory identification of open loop transfer functions in our formalism.

By the way, we should pay our attention to calculation of iteration procedure of Eq. (3). Though the matrix $E(n)$ is symmetric, there is a possibility to lose its symmetry in the generalized inverse calculation numerically. Then, symmetry of $E(n)$ is recovered in each step of iteration, otherwise we have a false solution which shows a drift movement without convergence to some direction in a subspace. In our formalism without symmetry process the drift phenomenon has been observed even in monotonic convergence cases. The symmetry procedure for $E(n)$ is not incorporated into our calculation, until we can have a thorn phenomenon.

As to HPF effects it is concluded that transfer functions are identified almost correctly in a high pass filter with lower than $5 \mathrm{~Hz}$ cutoff frequency, if the HPF is stable.

\section{References}

[1] K. Kishida: Modeling of feedback system and identification of transfer functions, Proc. of 30th ISCIE Intern. Symp. on Stochastic Systems Theory and Its Applications, (SSS30), 81/86 (1999).

[2] K. Kishida and K. Sato: Identification of transfer functions in a feedback system and stochastic inverse problem, Proc. of 31st ISCIE Intern. Symp. on Stochastic Systems Theory and Its Applications, (SSS31), 25/30 (2000).

[3] K.Kishida: Electroencephalography and Identification of Transfer Functions in a Feedback System", Proc. of 32nd ISCIE Intern. Symp. on Stochastic Systems Theory and Its Applications, (SSS32), $57 / 62$ (2001).

[4] K.Kishida: Identification Method based on Feedback System Theory and Stochastic Inverse Problems in Complex Systems", Proc. of ISM Symp. on Statistical Researches in Complex Systems, 65/79 (2001).

[5] K.Kishida: Contraction of Information and Its Inverse Problem in Reactor System Identification", In Advances in Nuclear Science and Technology, Lewins L. and Becker (eds.), vol.23, pp. 1-68, Plenum Press, N.Y. (1996).

[6] G. Goodwin, and R. L. Payne, Dynamic System Identification: Experiment Design and Data Analysis, Academic Press, (1977).

[7] K.Kishida: Contraction of Information in Systems far from Equilibrium, J. Math. Phys., 32, 92/98 (1991). 